\title{
Classifier learning and modality in a polyglot savant
}

\author{
Gary Morgan $^{\mathrm{a}, *}$, Neil Smith ${ }^{\mathrm{b}}$, Ianthi Tsimpli ${ }^{\mathrm{c}}$, Bencie Woll ${ }^{\mathrm{b}}$ \\ ${ }^{a}$ Department of Language \& Communication Science, City University, \\ Northampton Square, London EC1V OHB, UK \\ ${ }^{\mathrm{b}}$ University College, London, $U K$ \\ ${ }^{\mathrm{c}}$ Aristotle University of Thessaloniki, Greece
}

Received 1 April 2004; received in revised form 1 June 2004; accepted 1 November 2005

Available online 6 March 2006

\begin{abstract}
Christopher (C) is a mildly autistic, severely apraxic, savant who learns languages with surprising ease. Surprising because outside language-learning he has severe difficulties in 'non-verbal' cognitive domains, particularly where his visuo-spatial abilities are involved. C's unique language learning skill has been reported on in several previous studies, but his verbal abilities have previously been measured mainly in his learning of different spoken and written languages, that is in the modality of oral language. In earlier work we have also described some aspects of C's learning of British Sign Language (BSL), a language produced and perceived in the visual modality. The present investigation gives further details of C's learning of BSL, by focusing on his use of sign language classifiers, and compares his uneven learning with that of a group of university language honours students. Specifically, although after exposure to an introduction to sign language course, C performed within normal limits on a variety of BSL constructions, he had significant difficulty with the production and comprehension of classifiers, a category that crucially involves the interaction of grammatical and spatial cognition. Here we present two new sets of results of tests of C's understanding and use of classifiers: his comprehension of sentences with differing degrees of spatial and grammatical encoding, and his performance on a placement task involving classifiers. In the first task C's poor performance was worst on those sentences with the most complex demands on spatial cognition. In the task placing objects on a table $\mathrm{C}$ performed well with real object placement but could not use the classifier system to represent object placement in sign space. Performance on these tasks as well as C's uneven profile of BSL acquisition is interpreted within an established model of the mind but with modifications to accommodate the interface between language processing and non-verbal, visuo-spatial cognition.
\end{abstract}

(C) 2006 Elsevier B.V. All rights reserved.

Keywords: Savant; BSL; Classifiers; Spatial cognition; Modality

\footnotetext{
* Corresponding author. Tel.: +44 207040 8291; fax: +44 2070408577.

E-mail address: g.morgan@city.ac.uk (G. Morgan).
} 


\section{Christopher's linguistic and psychological profile}

Christopher (C) is a right-handed man (date of birth 6 January 1962) who combines a remarkable ability for learning new languages with serious disabilities in other domains. He is mildly autistic and severely apraxic; he lives in sheltered accommodation because he is unable to look after himself; he is unable to master noughts and crosses (tic-tac-toe), and he has some difficulty with conservation tasks, in particular conservation of number. Nonetheless, as documented extensively elsewhere (e.g. Smith and Tsimpli, 1995), his knowledge of English syntax is essentially normal. ${ }^{1}$

When tested on a range of constructions including passives, negatives, Wh-questions, relative clauses, stranded prepositions, parasitic gaps, agreement processes, and so on, he performed like any other native speaker. Moreover, his inferential abilities in the use of language also fall within normal limits. Most strikingly, he has a talent for learning 'second' languages: he can read, write, speak, understand and translate some 20 or more languages, including Danish, Dutch, Finnish, French, German, Modern Greek, Hindi, Italian, Norwegian, Polish, Portuguese, Russian, Spanish, Swedish, Turkish and Welsh. His ability ranges from fluency (in French, Greek and Polish) to mastery of the bare elements of the language (in Finnish, Norwegian and Welsh). In experiments in which we taught him new languages, specifically Berber and the invented language Epun (Smith et al., 1993; Smith and Tsimpli, 1995), he mastered a complex morphology with consummate ease and performed as well as or better than undergraduate controls.

Given this linguistic talent, it is not surprising that $\mathrm{C}$ scores well on standard measures of verbal intelligence, whereas on tests of non-verbal intelligence he scores relatively poorly (see Figs. 1 and 2; O'Connor and Hermelin, 1991; Smith and Tsimpli, 1995; Morgan et al., 2002a).

This asymmetry in his performance was corroborated by novel tests (based on Gollin Figures) in which he scored dramatically better on the identification of partial representations of words than on the partial representations of objects (Smith and Tsimpli, 1995:8ff.) It is important to note, however, that C's linguistic performance in his 'second' languages is not flawless. His mastery of the lexicon and morphology is remarkable and remarkably fast, but his mastery of syntax is far from perfect in that he seems to reach a plateau of achievement and thereafter appears to be influenced in his comprehension and production of foreign languages by the syntax of English, his first language.

It is also noteworthy that he has some difficulty with the non-English phonology of many of these languages. This is partly because he has acquired much of his knowledge on the basis of a written input, partly because he has a slight speech impediment, but mainly because his talent resides pre-eminently in the acquisition of morphology and the lexicon.

$\mathrm{C}$ has considerable difficulty in finding his way around, and has apraxic problems in tasks such as hanging a cup on a hook that require good hand-eye coordination. These deficits are corroborated by his results on tests of non-verbal intelligence which show that he has difficulty in the following domains:

- He finds the reproduction of spatial configurations difficult. For example, his recall of complex geometrical figures, e.g. the Rey-Osterrieth Complex Figure Test (Osterrieth, 1992) is

\footnotetext{
${ }^{1}$ There are one or two areas, e.g. topicalisation, where his judgement is abnormal. These cases are discussed extensively in the published references, see especially Tsimpli and Smith (1998).
} 
Standard verbal tests

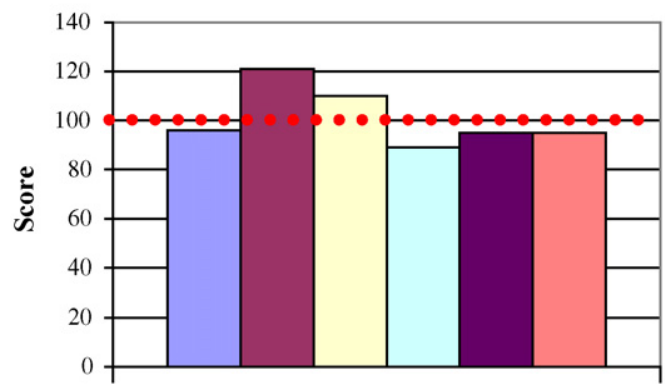

Test

Standard score (100)
口WISC-R, UK

$\square$ Peabody English

$\square$ Peabody German

$\square$ Peabody French

Peabody Spanish

Warrington word recognition

Fig. 1. Christopher's scores on standard verbal tests.

impaired. On the Goodenough Draw a Man Test he scored 40 and 63 (administered at ages 14 and 32) where a standard score is 100.

- The maintenance of spatial relations between objects on a page or in the real world while rotating or rearranging them mentally is impaired.

- The processing of non-linguistic information such as facial expression, is problematic.

The above outline makes it clear that $\mathrm{C}$ displays a marked asymmetry between his verbal skills in 'spoken' languages and his restricted non-verbal abilities. Asymmetries of one kind or another are characteristic, perhaps even definitional, of the 'savant syndrome' (to the extent that such a category exists), and many subjects on the autistic spectrum have obsessional tendencies-

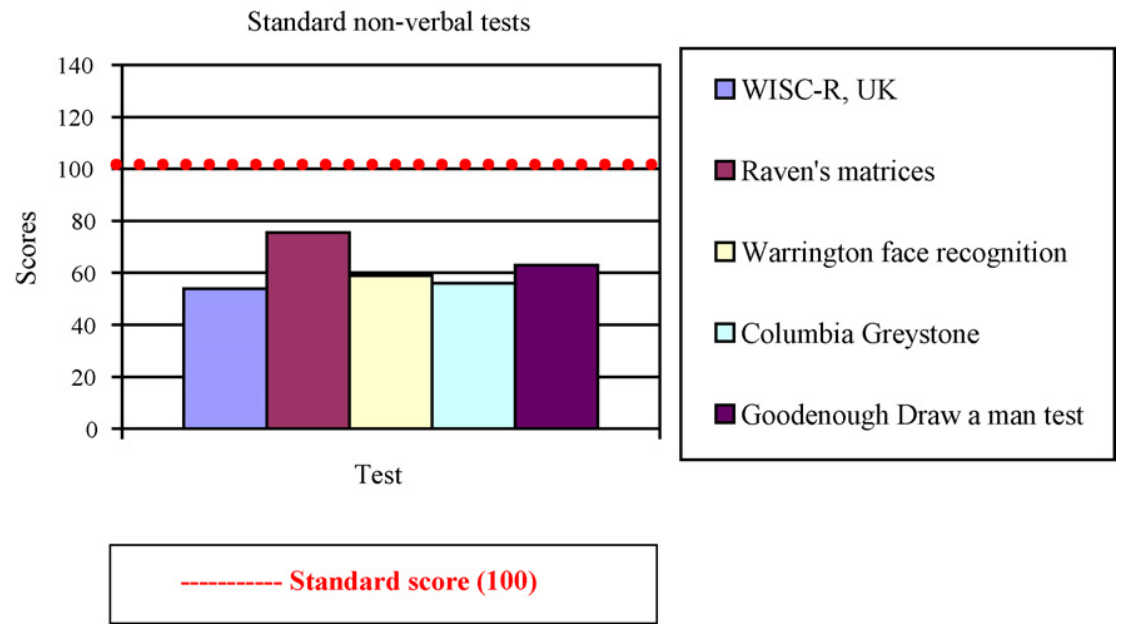

Fig. 2. Christopher's scores on standard non-verbal tests. 
tendencies that usually flourish at the expense of language. By contrast, C's obsession is learning new languages, and he spends most of his free time in pursuit of this interest-reading books and newspapers, interacting with native speakers of other languages, working through elementary grammars, and so on.

This combination of abilities and disabilities suggested a unique language learning experiment: teaching him BSL. This language offers him the linguistic features with which he is preoccupied, while presenting them through a modality with which he has great difficulty. One would expect that learning BSL would pose a significant problem for $\mathrm{C}$ because, despite its status as a fully-fledged natural language, its grammar makes crucial and systematic use of space.

\section{Space in BSL}

BSL (like all other sign languages) uses space in three different ways:

1. For phonological purposes.

2. To express morpho-syntactic relations.

3. To express spatial (topographic) relations.

\subsection{Phonology}

In all signed languages, signs are articulated in space and are made up of various handshapes in different locations, movements and orientations. Changing any one of these four parameters may change the meaning. This is most obvious in minimal pairs such as DRUNK and BLIND where handshape differs ('V sign' with fingers bent and 'V sign' with fingers straight) while location, movement and orientation are identical. In the pair AFTERNOON and NAME it is just the location of the signs (chin versus forehead) that distinguishes the two meanings. Thus the use of spatial contrast functions in the language to distinguish phonological categories (for more details see Van der Hulst, 1993; Brentari, 1998).

\subsection{Morpho-syntax}

Signers transmit information using a syntax which is spatially organised (see Janis, 1995). This role of sign space in BSL is the most obvious manifestation of the influence of modality. In (1) the articulation of the BSL verb TEASE moves between two abstract spatial locations which have been assigned indexes (IX) on the right and left sides of sign space (indicated by subscripted $\mathrm{R}$ and $\mathrm{L}$ ) in the previous sentence. The direction of morphological inflection carried on the verb's movement then encodes the syntactic subject and object. ${ }^{2}$

\footnotetext{
${ }^{2}$ Signed sentences that appear in the text follow standard notation conventions. Signs are represented by upper-case English glosses. 'IX' is a point to an area of sign space which acts as a syntactic index for referring to an argument in the sentence. Subscripted upper-case letters, R, L and C, indicate locations in sign-space, namely right, left and centre; subscripted lower-case letters indicate coindexation. Above the glosses, non-manual markers such as face and head movements are indicated by a horizontal line across the affected segment(s). A signed sentence which contains classifier constructions is glossed by first indicating the use of a classifier (CL) then the classifier handshape and lastly the predicate. This gloss is transcribed as one construction, e.g. CL- $\mathrm{G}_{\mathrm{C}}$ 'the pen is in the centre'-where $\mathrm{G}$ marks a long-thin object.
} 
(1)
$\mathrm{JOHN}_{\mathrm{a}}$
$\mathrm{IX}_{\mathrm{aL}}$
$\mathrm{ALISON}_{\mathrm{b}}$
$\mathrm{IX}_{\mathrm{bR}}$
${ }_{\mathrm{a}} \mathrm{TEASE}_{\mathrm{b}}$
'There is John and there is Alison, John teases Alison'

It is crucial to understand that the assignment of indexes to these positions is abstract because the signer is talking about non-present referents. If John and Alison were present (e.g. standing in front of the signer) the signer would use their real world locations as indexes.

Not all morpho-syntactic information is spatially conveyed: in other areas of BSL syntax, nonmanual markers (e.g. head shakes) rather than spatial contrasts are used to encode grammatical distinctions such as negation. An example is shown in (2):

\author{
ICE-CREAM EAT I \\ 'I don't eat ice-cream'
}

\title{
2.3. Spatial (topographic) relations
}

Emmorey et al. (1995) and Campbell and Woll (2003) characterise the topographic use of space as the exploitation of the space within which signs are articulated to describe the physical position and orientation of objects or people, with the spatial relations among signs corresponding in a topographic manner to actual relations among the objects described. Signed languages thus differ from spoken languages, not only because a visual medium is recruited for language, but additionally in that certain linguistic structures use spatial locations topographically. "The linguistic conventions used in this spatial mapping specify the position of objects in a highly geometric and non-arbitrary fashion by situating certain sign forms (e.g. classifiers) in space such that they maintain the topographic relations of the world-space being described." (Emmorey et al., 1995:43/44). As suggested in this quotation, the major linguistic constructions which appear to use space in their syntax involve noun classifiers and classifier predicates referring to space and motion.

\section{Classifiers}

It is not certain that the linguistic category referred to as 'classifier' in sign language research is exactly the same as that described in the grammars of some spoken languages (e.g. Aikhenvald, 2000; Grinevald, 2000). However, it is uncontroversial that signers use them systematically to talk about space. In section 6 we discuss briefly whether C's difficulty with classifiers should be attributed to his lack of familiarity with the linguistic category or to the modality in which that category is expressed; in this section we discuss the role of classifiers in the expression of spatial information.

Classifiers describe spatial relations in terms of a correspondence between real-world entities and an array represented in sign space as a spatial map. An example of this spatial function of classifiers is given in (3), where the signer is describing the relative locations of three objects on a table. The handshape '5-claw' is a variant of ' 5 ' with fingers curved and is used to indicate objects with height and width, e.g. houses, boxes or bunches of keys. 

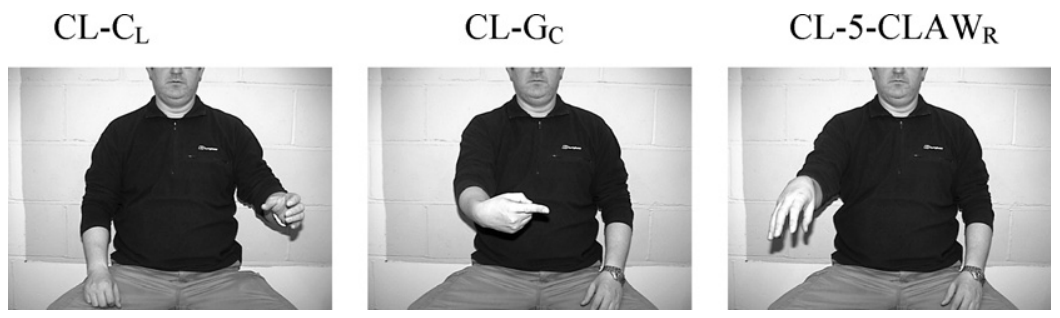

Fig. 3. Classifiers in sign space when describing the sentence in (3).

TABLE CUP CL- $\mathrm{C}_{\mathrm{L}}$ PEN CL-G $\mathrm{C}$ KEY CL-5-CLAW $\mathrm{R}_{\mathrm{H}}$

'On the table there is a cup on the left, a pen in the middle and a bunch of keys on the right'

The signer first signs TABLE, then CUP followed by a classifier handshape (C handshape), which is placed at a location corresponding to a map of the real world layout being described. The signer then signs PEN, followed by a classifier sign ( $G$ handshape) which is placed to the right of the first classifier. ${ }^{3}$ The last object KEY is signed, followed by a classifier (5-Claw) representing 'bunch of keys' articulated on the right side of sign space. For each item, the signer places the classifier signs in sign space in correspondence with their real world location. This is shown in Fig. 3.

Emmorey (1996) has suggested that, unlike spoken languages, signed languages necessarily involve an intimate connection between the linguistic system and the spatial cognitive system. More recently, she has described this as 'talking about space with space' (Emmorey and Falgier, 1999:3; see also Emmorey, 2003). The influence of spatial cognition on the processing of classifiers, albeit indirect-i.e. through language, is then supposedly greater in signed languages than in spoken languages. We hope to contribute to the understanding of this linguistic and cognitive domain.

\section{A language learning experiment}

In learning BSL, $\mathrm{C}$ was exposed to the typical first course in BSL which ran for 8 months and included $24 \mathrm{~h}$ of instruction in both taught and conversational modes. C also had access to BSL books and videos which he studied between classes. All his exposure to BSL came from native adult signers. We compared C's learning with a group of 40 (30 female, 10 male) hearing University students, aged between 18 and 30 years. All had progressed to post A-level in either French, German or Spanish, but were not native speakers of these languages. ${ }^{4}$ All were BSL novices. We attempted to make the input to $\mathrm{C}$ and the controls as similar as was feasible. For further details, see Morgan et al., 2002a.

The contents of the first course both $\mathrm{C}$ and the comparator group followed included the following:

\footnotetext{
${ }^{3}$ This gloss is transcribed as one construction, e.g. CL- $\mathrm{G}_{\mathrm{C}}$ 'the pen is in the centre'-where G marks a long-thin object.

${ }^{4}$ This marks a level of attainment sufficient for starting a University degree in the literature of that language in the UK.
} 
1. Introduction to signing. Naming, questions and finger-spelling.

2. Wh and yes-no questions. Simple naming questions, asking about work, family, foods. Vocabulary and practice.

3. Negation markers in different syntactic constructions.

4. Verb agreement through different morphological modifications of the sign. Vocabulary and practice.

5. Using classifiers and sign space. Using facial expression.

6. Review of previous topics and practice.

7. Narratives with two characters, classifiers, sign space and role shift.

8. Using topic markers in simple sentences, simple verb aspect markers.

9. Question and negation with new verbs, finger-spelling.

10. Verb agreement with locations in sign space.

11. Narrative with three characters.

12. Complex sentences.

In the first test of comprehension of negatives (referred to as 'NEG' below) we tested both $\mathrm{C}$ and the comparator group on video-taped signed utterances with and without negative elements. The task was to pick the appropriate picture, e.g. 'the dog without the bone' from a set of four possible alternatives after seeing a signed sentence. In the second negation test (NEG*) we constructed a grammaticality judgement task involving signed sentences with negation on the verb, where half of the sentences involved ungrammatical combinations of negative marker and manual sign, e.g. EAT-NOT. We administered two tests of verb agreement comprehension: in the first (labelled AGR below) subjects were shown signed sentences on video. All the sentences had a verb which moves in BSL to show agreement with the subject and direct object, as in example (1) above. Each signed sentence had two referents (e.g. 'John' and 'Alison'). After each signed utterance, subjects picked that written English sentence which constituted the more appropriate translation. There were two sentences to choose from, which varied the role the referents took as subject or direct object, e.g. John did the teasing or Alison did the teasing. The test labelled AGR* below measured subjects' ability to judge whether verbs in different sentences were able to encode person agreement through movement between indexed locations. Agreement verbs such as ASK, GIVE or TEASE, as in example (1) can move between spatial syntactic locations to encode person agreement but other verbs cannot, e.g. LIKE, KNOW or WANT (Padden, 1988). Subjects viewed a signed sentence with a grammatical or ungrammatical use of verb agreement along with an English translation. They were asked to judge whether the BSL sentence 'was well formed or not'.

The other tests involved the comprehension of sentences with classifiers. The first test (CL TRAN) required subjects to watch 10 signed sentences involving a classifier and then choose one of three written English sentences. For example, in one item the BSL target was 'a line of telephones' produced with a Y hand shape articulated several times in a straight line in sign space. The choices were:

1. a line of horses;

2. a line of cars;

3. a line of telephones.

In the second test (CL PIC) subjects watched 10 signed sentences and then picked a corresponding picture from four picture alternatives. 


\section{Results}

\subsection{General learning}

C learned BSL to a level comparable to that of a normal learner, though in specific areas of the language his comprehension and production showed systematic asymmetries. We have reported elsewhere (Morgan et al., 2002a,b) on his proficient mastery, both in production and in comprehension, of single signs, showing that despite deficits in hand-eye co-ordination, he has acquired the basic parameters of sign formation sufficiently to underpin a good working vocabulary. Across the grammar of BSL, C's and the control group's performance is shown in Fig. 4.

In conversation $\mathrm{C}$ used negation appropriately although he had difficulty in combining the use of non-manual and manual signs. In both NEG tests (shown in Fig. 4), there were no significant differences in performance between $\mathrm{C}$ and the controls. In the domain of verb agreement, $\mathrm{C}$ used inflections towards the locations of present referents more easily and productively than with abstract locations in sign space. In both AGR tests (again, shown in Fig. 4) C's understanding of verb agreement was within normal learner limits although closer to the lower-scoring learners in the control group. However, in contrast to his superlative performance with verb agreement morphology in his learning of spoken languages - Berber, for instance - his learning of BSL agreement was poor. It has been suggested that verb agreement in sign languages necessarily involves the representation of real world spatial mappings (e.g. Liddell, 2000; Lillo-Martin, 2002). C's performance adds further support to the implication of this suggestion that verb agreement in BSL is not completely identical to that in spoken languages.

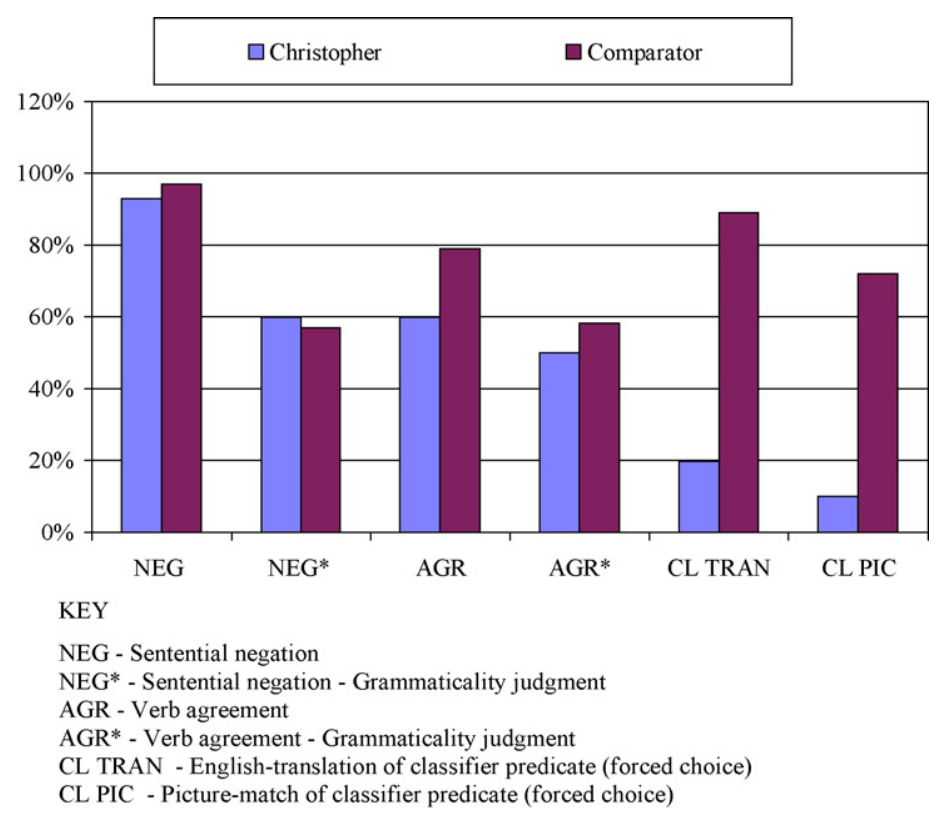

Fig. 4. Assessments of comprehension across BSL grammar tests: Christopher and mean comparator scores. 


\subsection{Learning classifiers}

The one area where $\mathrm{C}$ performed significantly worse than the control group was with classifiers. In the CL TRAN test (matching a signed sentence to a written English translation) $\mathrm{C}$ scored $20 \%$ correct (chance was 33\%), whereas the scores of the control group were between $80 \%$ and $100 \%$ (mean $89 \%$, S.D. $=9.9 \%$ ). In the CL PIC test (matching a signed sentence to a picture) $\mathrm{C}$ scored $10 \%$ correct (chance was 25\%), whereas the controls scored between $50 \%$ and $100 \%$ (mean $72 \%$, S.D. $=13.8 \%$ ). C's performance on both tests was therefore extremely poor: below three standard deviations of the mean.

In both of these tests an interesting asymmetry appeared. $\mathrm{C}$ typically made errors which showed that he had correctly identified the main lexical items (on the basis of the handshapes) but made mistakes with regard to the location of the objects recognised. For instance, after seeing the sign sequence CL-Bent-B-BOOK-ON-CL-B-BED which translated as 'a book on a bed', C chose the picture of 'a book under a bed', rather than either of the other pictures which showed 'a ball under a chair' and 'a comb on a bed'. Thus, it seems that the cause of his poor performance was that he made errors in identifying the spatial relation expressed with the classifier even when he had correctly identified the lexical information expressed in the sign: e.g. 'flat object' versus 'round object' (viz. a book versus a ball).

It is striking that C's performance showed a systematic asymmetry between classifiers and other aspects of BSL, whilst that of the controls was consistent across these different domains (as shown in Fig. 4). A possible explanation for C's pattern of behaviour is that it reflects a dissociation between the various cognitive components - linguistic and spatial - involved in the representation of classifiers; in particular, we suspect that $\mathrm{C}$ has problems with features that draw on spatial cognition, an area in which it is independently known that he has a severe deficit.

It may also be relevant to this hypothesis that C's performance on agreement in BSL is significantly worse than his excellent mastery of morphological agreement in his spoken 'second' languages (Smith and Tsimpli, 1995). That is, it seems that the signed modality inhibits some aspects of C's otherwise enhanced language learning abilities. Moreover, the control group's performance on tests of verb agreement was similar to C's, suggesting that his superiority to ordinary L2 learners is limited to spoken (especially written) languages. C's relatively poor performance on spatial aspects of BSL, in particular classifiers encoding topographic relations, suggests that the classifier system is crucially interacting with spatial cognition, and that it is this which is causing $\mathrm{C}$ difficulties.

\section{Grammatical versus spatial relations}

As suggested in section 3, there are in fact two possible sources for C's difficulty with classifiers. The first has to do with his spatial deficit, the second with his lack of L1 or L2 experience with languages involving a classifier system for encoding grammatical relations. In order to tease apart these alternatives (space versus grammar) we devised 52 signed sentences, half (26) of which involved classifiers in different grammatical constructions (the predicate encoded plurality, aspect, movement and manner distinctions, etc.) but with little explicit spatial information. An example is shown in (4), in which the verb plus classifier handshape encodes the path of the person's movement, but does not specify any further spatial relations. The other 26 sentences included classifiers which did encode topographical information about spatial relations in sign space: an example is shown in (5): 


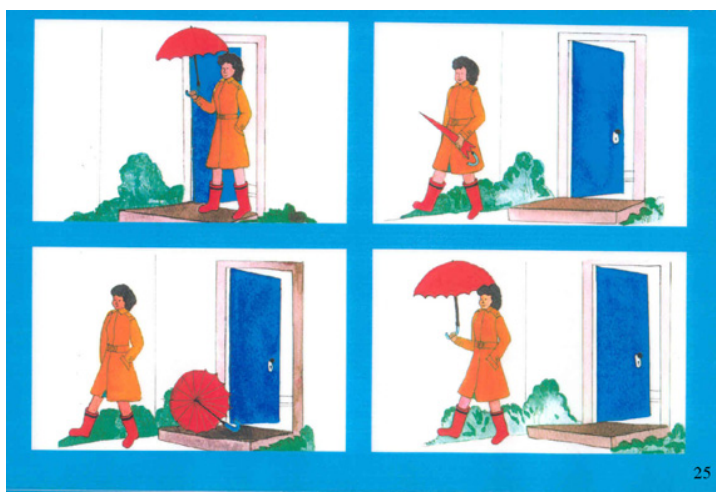

Fig. 5. WOMAN UMBRELLA CL-G-PERSON-WALK (bottom right picture is correct).

\author{
WOMAN UMBRELLA CL-G-PERSON-WALK \\ 'the woman with the umbrella walked along' \\ HOUSE CL-5-CLAW-HOUSE ${ }_{R}$ \\ 'the house was over on the far right'
}

Taken as a whole the example sentences included some which were weighted more towards grammatical relations and others that had more spatial information in their articulation. Each of the 52 sentences corresponded to a picture stimulus, and the 'grammar' and 'space' examples were randomly ordered. $\mathrm{C}$ was asked to watch the signed sentence and select the corresponding picture from a choice of four alternatives. The corresponding test pictures which were used with sentences (4) and (5) are shown in Figs. 5 and 6.

He identified 31 of the 52 classifiers correctly $(59.6 \%$; chance $=25 \%)$, but with an interesting asymmetry: selecting the correct picture for $11(42 \%$; chance $=25 \%)$ of the 26 spatial relation sentences, and for $20(77 \%$; chance $=25 \%)$ of the 26 sentences involving classifiers marking grammatical functions.

The distinction between 'grammatical' and 'spatial' functions of signing space in these sentences is not always entirely clear-cut. Because there exists a continuum between spatial and non-spatial uses of sign space (Emmorey, 2002) all the sentences involve some features of both, albeit to different degrees. Nevertheless, we suggest that C's differential comprehension of classifiers relates to properties that make the 'spatial' sentences representationally distinct from 'grammatical' ones. 5

Specifically, the 'spatial' sentence in (5) indicates the static location of the argument (the house) in a particular place in relation to other aspects of the picture, whereas the 'grammatical' example in (4) includes more arbitrary linguistic information, i.e. woman, umbrella and movement (walk). To arrive at an appropriate interpretation of (5) one needs to decode both the lexical representation of the concept 'house' and the spatio-syntactic mapping of the topographic location. This requirement would appear to be very similar to the cognition required to recall where a particular part of the Rey-Osterrieth complex figure is located.

${ }^{5}$ A final decision about the source of C's difficulties must await his learning of spoken languages involving classifiers. 


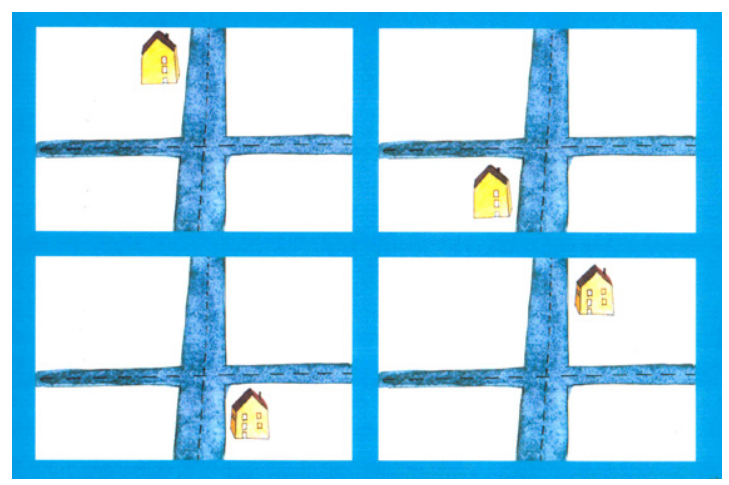

Fig. 6. HOUSE CL-5-CLAW-HOUSE $\mathrm{R}_{\mathrm{R}}$ (bottom right picture is correct).

To arrive at a plausible interpretation of (4) on the other hand, involves decoding more lexical and syntactic information but, crucially, no spatial relations (except the movement of the subject in the predicate). A learner with only partial knowledge of BSL syntax but good mastery of the lexicon could assign an interpretation to both (4) and (5) by mapping purely lexical information onto a predicate-argument structure. However, a learner with only partial knowledge of BSL syntax and a cognitive deficit in manipulating spatial relations (where in space the referent is located), would be expected to show differential performance on these two types of sentence.

\section{1. 'Setting the table' with classifiers}

We followed the comparison of C's performance on grammatical versus spatial information with a second test, which we called 'setting the table'. This activity is common in the teaching of BSL at this stage, where students have to sign the position of objects and people in relation to each other using classifier handshapes in sign space. The task focused on C's understanding and use of classifiers to describe different (spatial) layouts of two, three or four objects on a table of the kind shown in example (3). We report on two results from this study. In the test $\mathrm{C}$ sat either beside his sign teacher or facing her. In the first set of 15 trials the teacher placed the objects in a specific spatial configuration in front of her on a table. In the second set of trials she signed using the appropriate classifiers in an array of the same objects in front of her in sign space. The objects were a book, a cup, a bunch of keys and a pen (represented by B, C, 5-claw, and G handshapes, respectively). $\mathrm{C}$ had identical objects in front of him. Once the experimenter had finished either arranging the objects or signing them, $\mathrm{C}$ was asked to copy the arrangement using his set of objects. In the second trial he was explicitly told to sign his response rather than simply place the objects in position. ${ }^{6}$

In these two tests $\mathrm{C}$ performed much better in copying object placement than copying a signed sentence. The objects the experimenter had placed were in full view during C's copy. He scored $100 \%$ correct (15 out of 15) when seated next to the teacher and 93\% (14/15) correct when facing her. His one mistake when facing the teacher was to reverse an 'on-top-of' relation to 'underneath'. This excellent performance with real objects, contrasts drastically with his ability to copy the teacher's signed instructions to set the table. In contrast to the previous condition,

\footnotetext{
${ }^{6}$ Note that $\mathrm{C}$ had performed extremely well in tests using spoken language which demanded comparable abilities: see Smith and Tsimpli (1995):176f.
} 
when the signer signed the object locations they were momentarily 'present' in the sign space but then disappeared before the next sentence was signed. He scored $20 \%$ correct ( 3 out of 15) when seated next to the teacher and $0 \%$ when facing her (when facing the signer $\mathrm{C}$ was required to mentally reverse the placement of the classifier handshapes so as to maintain the original point of view). During testing, when the experimenter used a classifier predicate, $\mathrm{C}$ continually reached out to grasp and place a nearby object before the instructions were finished, despite being instructed not to do so. The task involving the placement of real objects is less demanding because it does not involve recalling spatial relations mapped onto classifiers by encoding a linguistic representation of the spatial arrangement. C's difficulties were with being able to hold in working memory a representation of the spatial arrangement of the linguistic labels vis-à-vis real world objects.

\section{Modality and language: a model of the mind}

In Smith and Tsimpli (1995:170), we developed a 'model of the mind', which attempted to incorporate aspects of Fodor's (1983) modularity thesis, Anderson's (1992) theory of intelligence, and our own hypotheses about 'quasi-modules' (Tsimpli and Smith, 1998). A crucial part of Anderson's theory is the postulation of two different 'Specific Processors' dedicated to the manipulation of visuo-spatial and verbal-propositional representations provided by auditory, visual and linguistic input systems. Part of our account accordingly suggested that the Language of Thought (LoT, see Fodor, 1975) was fed by representations provided by either of these 'Specific Processors'. We further suggested that "it is easier to express in natural language something which is closer to isomorphic with the language of thought, and we take it as axiomatic that verbal representations are closer to the language of thought than spatial ones are" (Smith and Tsimpli, 1995:167). This, of course, was written some years before we embarked on the project of teaching C BSL, and we wish to elaborate our proposal in the light of this research.

There has recently been a re-emergence of interest in a version of the Sapir-Whorf hypothesis (see, e.g. Davidoff, 2001; Spelke and Tsivkin, 2001, and other contributions in the same volume). We think that the properties of sign language and C's learning of it provide further evidence. We should emphasize that we do not subscribe to the strong form of the SapirWhorf hypothesis, but that the use of particular systems - auditory or visual - should facilitate the representation of some messages over others seems both plausible and unexceptionable. That is, for someone who is bilingual in a signed and a spoken language, mapping a spatial array into the signed language should be simpler than mapping it into the spoken language, whereas mapping (for instance) a uni-dimensional representation such as time into the spoken or the signed language should be equally easy, and (perhaps) mapping a representation with (onomatopoeic) auditory properties such as relative loudness should be easier into the spoken than into the signed language. This is not to deny that spatial features have become part of the grammar in signed languages and are not just borrowed directly from cognition. The representation of spatial arrays in both signed and spoken languages presupposes the ability to represent spatial relations abstractly. But the signed versus spoken modality in the representation of spatial arrays gives rise to different possibilities of compensation for the deficit in spatial cognition. In C's case, his intact spoken language abilities enable him to represent spatial arrays linguistically. Signed languages, on the other hand, which integrate spatial relations into a linguistic system embedded in the spatial modality, mean that a spatial deficit is difficult to compensate for. 


\begin{tabular}{|cccc|}
\hline $\begin{array}{c}\text { Linguistic processing } \\
\text { Negation }\end{array}$ & Verb agreement & $>$ & $\begin{array}{c}\text { Conflation of spatial } \\
\text { and linguistic processing } \\
\text { Classifers }\end{array}$ \\
\hline
\end{tabular}

Fig. 7. Spectrum of involvement of visuo-spatial cognition in different aspects of BSL grammar .

On the basis of the discussion above, we think that it is justified to assume that BSL has recourse to linguistic structures with greater or less involvement of visuo-spatial mapping. This is represented in the hierarchy in Fig. 7, where classifiers are seen as involving both purely syntactic and visuo-spatial mapping functions: they are at the interface between syntax and space.

In light of the results for $\mathrm{C}$ and the control group on classifiers we can now elaborate on the model of the mind proposed earlier. Following Anderson (1992) we assume that it is necessary to postulate at least the following components of the mind: a Basic Processing Mechanism, Specific Processors dedicated to processing visuo-spatial and verbal-propositional stimuli, and a set of Fodorian modules or input systems. To this we added a set of quasi-modules (Tsimpli and Smith, 1998) whose responsibility was to process domain-specific material from such areas as music, Theory of Mind and, in part, language. Quasi-modules are distinct from Fodorian modules both in their lack of informational encapsulation and in operating over a conceptual rather than perceptual vocabulary. We now think, following Van der Zee and Nikanne (2000b:2), that it is necessary to add to this array additional components for spatial structure and motor structure. They propose a schematic configuration with three interfaces like that in (6):

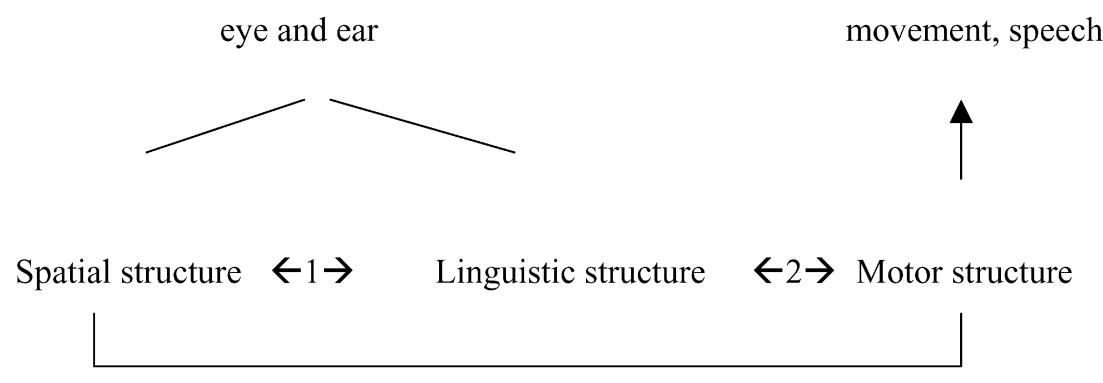

where Interface 1 maps spatial information to linguistic information and vice versa; Interface 2 maps linguistic information to motoric information and vice versa; and Interface 3 maps spatial information into motoric information and vice versa. We are aware of a variety of other complications, some of which are spelled out in work by Jackendoff (1983), Nikanne (2000) and Platzack (2000), but this is sufficient for present purposes.

C's uneven performance in his learning of BSL would then be related to defects in the spatial and motor components of this model. More specifically, his asymmetric success with classifiers is a function of problems at the interfaces between the spatial and linguistic modules, and the linguistic and motor modules. To the extent that this compartmentalised structure allows the appropriate dissociations to be described it provides support for the model we are putting forward. In work in progress we outline the detailed workings of each of the components for each of C's activities (Morgan et al., in preparation). 


\section{Conclusions}

$\mathrm{C}$ has not learned BSL with the same ease as his other second languages. We have argued that part of this differential success is a direct result of deficits in his spatial cognition or the interaction of spatial cognition and language. A comparison of different areas of BSL suggests that spatial cognition may play more or less of a role in different linguistic constructions. We have presented results from his learning of three areas of BSL. First, negation does not exploit space to any real degree and consequently caused no real problems for C. Second, although verb agreement is based in a spatial syntax it appears less vulnerable to C's spatial deficit than classifier constructions. In spontaneous signing $\mathrm{C}$ used verb agreement morphology with locations indexed by present referents, and in tests of his understanding of abstract indexes he performed poorly but within normal learner limits. Because $\mathrm{C}$ normally excels in using morphology in his other second languages his mediocre performance with BSL verb agreement morphology suggests that his deficit in spatial cognition is impinging on his learning. Third, while $\mathrm{C}$ had some success identifying the referents encoded in the classifier handshape, he performed worse than any of the control group in all aspects of classifier use and comprehension in movement and location predicates.

We had argued previously (Smith and Tsimpli, 1995:39) that C may have difficulty in accessing some components of the Central System - e.g. the system of spatial cognition - during language processing. In the present study we have found the same kind of interactional difficulty in his use of BSL classifiers and spatial mapping. Our results suggest that there is a fundamental modality-dependent difference for $\mathrm{C}$ in the processing and learning of a second language. Teaching BSL to $\mathrm{C}$ has revealed the importance of modality in his language learning. Compared with typical learners with intact spatial cognition systems who are confronted with the linguistic aspects of BSL, $\mathrm{C}$ is at a disadvantage. By examining the learning of classifiers in BSL, we hope to have shown both that a spatial mapping deficit reveals the importance of intact spatial cognition in sign language and especially classifier processing, and to have provided evidence for a particular model of the mind.

\section{Acknowledgments}

We are grateful to John Carlile, Ann Law, Anne Sturdy and Frances Elton for advice and information. We are particularly indebted to the Leverhulme Trust, which has supported our work with $\mathrm{C}$ over many years under grant F 134.

\section{References}

Aikhenvald, A.Y., 2000. Classifiers: A Typology of Noun Categorization Devices. OUP, Oxford.

Anderson, M., 1992. Intelligence and Development: A Cognitive Theory. Blackwell, Oxford.

Brentari, D., 1998. A Prosodic Model of Sign Language Phonology. MIT press, Cambridge, MA.

Campbell, R., Woll, B., 2003. Space is special in sign. Trends in Cognitive Sciences 7 (1), 5-7.

Davidoff, J., 2001. Language and perceptual categorisation. Trends in Cognitive Sciences 5, 382-387.

Emmorey, K., 1996. The confluence of space and language in signed languages. In: Bloom, P., et al. (Eds.), Language and space. MIT Press, Cambridge, MA, pp. 171-209.

Emmorey, K., 2002. Language, Cognition and The Brain: Insights from Sign Language Research. Lawrence Erlbaum Associates, Mahwah NJ.

Emmorey, K., 2003. Perspectives on Classifier Constructions in Sign Language. Erlbaum, Mahwah, NJ. 
Emmorey, K., Corina, D., Bellugi, U., 1995. Differential processing of topographic and referential functions of space. In: Emmorey, K., Reilly, J. (Eds.), Language, Gesture, and Space. Erlbaum, Hillsdale, NJ, pp. 43-62.

Emmorey, K., Falgier, B., 1999. Talking about space with space: describing environments in ASL. In: Winston, E. (Ed.), Story telling and conversation: discourse in deaf communities. Gallaudet University Press, Washington, DC, pp. 3-26.

Fodor, J., 1975. The Language of Thought. Harvard University Press, Cambridge, MA.

Fodor, J., 1983. The Modularity of Mind. MIT Press, Cambridge, MA.

Grinevald, C., 2000. A morphosyntactic typology of classifiers. In: Senft, G. (Ed.), Systems of Nominal Classification. CUP, Cambridge, pp. 50-92.

Jackendoff, R., 1983. Semantics and Cognition. MIT Press, Cambridge, MA.

Janis, W., 1995. A crosslinguistic perspective on ASL verb agreement. In: Emmorey, K., Reilly, J. (Eds.), Language, Gesture, and Space. Erlbaum, Hillsdale, NJ, pp. 195-223.

Liddell, S., 2000. Indicating verbs and pronouns: pointing away from agreement. In: Lane, K., Emmorey, K. (Eds.), The signs of Language Revisited: An Anthology to Honour Ursula Bellugi and Edward Klima. Lawrence Erlbaum Associates, Mahwah, NJ, pp. 303-320.

Lillo-Martin, D., 2002. Where are all the modality effects? In: Meier, R.P., Cormier, K., Quinto-Pozos, D. (Eds.), Modality and Structure in Signed and Spoken Language. Cambridge University Press, Cambridge, pp. $241-262$.

Morgan, G., Smith, N.V., Tsimpli, I.-M., Woll, B., 2002a. Language against the odds: the learning of British Sign Language by a polyglot savant. Journal of Linguistics $38,1-41$.

Morgan, G., Smith, N.V., Tsimpli, I.-M., Woll, B., 2002b. The effects of modality on BSL development in an exceptional learner. In: Meier, R.P., Cormier, K., Quinto-Pozos, D. (Eds.), Modality and Structure in Signed and Spoken Language. Cambridge University Press, Cambridge, pp. 422-441.

Morgan, G., Smith, N.V., Tsimpli, I.-M., Woll, B., in preparation. The signs of a savant, CUP.

Nikanne, U., 2000. In: Van der Zee, E., Nikanne, U. (Eds.), Some Restrictions in Linguistic Expressions of Spatial Movement, pp. 77-93.

O’Connor, N., Hermelin, B., 1991. A specific linguistic ability. American Journal of Mental Retardation 95, 673-680.

Osterrieth, P.A., 1992. The Complex Figure Copy Test (Archives of Psychology, 30, 206-353). Translation. The Clinical Neuropsychologist, 7, 3-21.

Padden, C., 1988. Interaction of Morphology and Syntax in American Sign Language: Outstanding Dissertations in Linguistics. Garland, New York.

Platzack, C., 2000. In: Van der Zee, E., Nikanne, U. (Eds.), Multiple Interfaces, pp. 21-53.

Smith, N.V., Tsimpli, I.-M., 1995. The Mind of a Savant: Language Learning and Modularity. Blackwell, Oxford.

Smith, N.V., Tsimpli, I.-M., Ouhalla, J., 1993. Learning the impossible: the acquisition of possible and impossible languages by a polyglot savant. Lingua $91,279-347$.

Spelke, E., Tsivkin, S., 2001. Initial knowledge and conceptual change: space and number. In: Bowerman, M., Levinson, S. (Eds.), Language Acquisition and Conceptual Development. CUP, Cambridge, pp. 70-97.

Tsimpli, I.-M., Smith, N.V., 1998. Modules and quasi-modules: language and theory of mind in a polyglot savant. Learning and Individual Differences 10, 193-215.

Van der Hulst, H., 1993. Units in the analysis of signs. Phonology 10 (2), 209-241.

Van der Zee, E., Nikanne, U., 2000b. In: Van der Zee, E., Nikanne, U. (Eds.), Introducing Cognitive Interfaces and Constraints on Linking Cognitive Information, pp. 1-17. 